\title{
Kommission Hüftsonografie SGUM
}

Die vermehrt durchgeführten Refresherkurse konnten die diesjährige Welle der Rezertifizierungs-Pflichtigen gut auffangen. Relevante Neuigkeiten sind nicht zu vermelden, abgesehen von der angepassten und vereinheitlichen Gebührenordnung. Für „Einwanderer“-Kollegen wurden ebenfalls Richtlinien zum Erhalt des Schweizerischen Fähigkeitsausweises
„Hüftsonografie“ publiziert, welche den unterschiedlichen Vorkenntnissen Rechnung tragen sollen. Die entsprechenden Einzelheiten sind unter „www.babyschall. ch“ unter der Rubrik „für Ärzte“ aufgeschaltet.

\author{
Beat Dubs \\ Präsident Kommission Hüftsonografie \\ SGUM
}

Die Studie über die Relevanz des Seitenvergleichs bei paarigen Extremitäten-Untersuchungen ist abgeschlossen, es sind fast 2000 Protokolle eingegangen, welche nun ausgewertet werden. Die Resultate werden am Dreiländertreffen im September in Davos vorgestellt und anschliessend publiziert.

Die üblichen Fragen von Leistungserbringern, aber auch Kostenträgern, betreffen vor allem unklare Interpretationen, Kumulationsverbote aber auch teilweise fehlerhafte Tarifanwendungen. Nicht alle Validatoren scheinen korrekt eingestellt zu sein, so werden mehrfach Kumulationsverbote zwischen Grundleistungen und Leistungen aus dem Kapitel 39 (Bildgebende Verfahren) gemeldet. Dieses Kumulationsverbot gilt nur für Inhaber des Facharzttitels Radiologie FMH, welche anstelle der Grundleistungen die sog. „Betriebsstelle“ abrechnen dürfen. Wenn hingegen Nichtradiologen an Spitälern
Grundleistungen abrechnen wollen und der Validator auf „Radiologie“ eingestellt ist, kommen solche Meldungen zwangsläufig vor. Ich empfehle deshalb in all diesen Fällen zuerst die korrekte Einstellung des Validators zu überprüfen, bevor ich mit Anfragen angegangen werde.

Seit der Inkraftsetzung der Tarmed-Version 1.08 ist vor allem das Kapitel 29 „Schmerztherapie“ aktuell geworden. Dieses Kapitel enthält eine Position 29.2200 für die Ultraschall-gesteuerte diagnostische/therapeutische Intervention. Prima vista ist sie wesentlich besser dotiert als die entsprechende Position 39.3710 im Kapitel „Bildgebende Verfahren“. Allerdings sind folgende Faktoren zu beachten:

- Für die Position 29.2200 ist der Besitz des Fähigkeitsausweises „Schmerztherapie“ erforderlich (den man aktuell allerdings relativ einfach durch Besitzstandserklärung erhalten kann).
- Diese Position 29.2200 ist der Sparte „Interventionelle Schmerztherapie“ zugeordnet, d.h. eine Kumulation mit der Position 39.0020/21 („AIP“) und 39.3800 (technische Grundleistung) ist nicht möglich, hingegen bei der Pos. 39.3710 schon. Somit relativiert sich die auf den ersten Blick grosse Differenz auf ein paar wenige Taxpunkte.

\section{Beat Dubs}

Leiter Taskforce Tarmed SGUM 